\title{
Genevieve Artigas-Menant, Lumières clandestines. Les papiers de Thomas Pinchon - Du secret des clandestins à la propagande voltairienne
}

\section{Nadia Minerva}

\section{(2) OpenEdition \\ Journals}

Edizione digitale

URL: https://journals.openedition.org/studifrancesi/40667

DOI: $10.4000 /$ studifrancesi.40667

ISSN: 2421-5856

\section{Editore}

Rosenberg \& Sellier

\section{Edizione cartacea}

Data di pubblicazione: 1 juillet 2004

Paginazione: 187-188

ISSN: 0039-2944

\section{Notizia bibliografica digitale}

Nadia Minerva, «Genevieve Artigas-Menant, Lumières clandestines. Les papiers de Thomas Pinchon - Du secret des clandestins à la propagande voltairienne», Studi Francesi [Online], 142 (XLVIII | I) | 2004, online dal 30 novembre 2015, consultato il 09 septembre 2021. URL: http://journals.openedition.org/ studifrancesi/40667; DOI: https://doi.org/10.4000/studifrancesi.40667

Questo documento è stato generato automaticamente il 9 septembre 2021.

\section{cc) $(8)$}

Studi Francesi è distribuita con Licenza Creative Commons Attribuzione - Non commerciale - Non opere derivate 4.0 Internazionale. 


\title{
Genevieve Artigas-Menant, Lumières clandestines. Les papiers de Thomas Pinchon - Du secret des clandestins à la propagande voltairienne
}

\author{
Nadia Minerva
}

\section{NOTIZIA}

GENEVIEVE ARTIGAS-MENANT, Lumières clandestines. Les papiers de Thomas Pinchon, Paris, Champion, 2001, pp. 425.

GENEVIEVE ARTIGAS-MENANT, Du secret des clandestins à la propagande voltairienne, Paris, Champion, 2001, pp. 440.

1 La collana «Libre pensée et littérature clandestine» ospita due volumi della nota ricercatrice che dirige l'équipe dell'«Inventaire des manuscrits philosophiques clandestins». I due testi sono complementari: il primo costituisce lo studio di un «caso», nel secondo il «caso Pinchon» è il polo di attrazione attorno al quale ruotano riflessioni su aspetti metodologici della ricerca relativa al corpus clandestino, analisi di autori che hanno alimentato questo fenomeno filosofico-letterario, presentazioni dei contenuti e della circolazione di opere che, per la loro dispersione, formano ancora oggi una galassia remota e incommensurabile.

Lumières clandestines è l'analisi di un nuovo insieme di testi destinati ad essere, secondo l'autrice una pietra miliare del settore: un fondo della biblioteca municipale di Vire, costituito dalla totalità delle carte personali di Pinchon (1700-1781), da lui stesso riordinate e destinate alla biblioteca della sua città natale: lettere e modelli di lettere, lista dei conoscenti e testimonianze sui suoi contemporanei, note di lettura e ricette... e, soprattutto, cinquanta manoscritti filosofici clandestini. Proprio per la sua organicità e ricchezza, il materiale rinvenuto getta nuova luce sull'insieme del fenomeno. Assiduo 
lettore della produzione dei Lumi, Pinchon è un personaggio singolare: grande viaggiatore, avventuriero nel Nuovo Mondo, seduttore di successo il cui nome è legato, durante il soggiomo londinese, a quello di Marie Leprince de Beaumont, agente segreto abilissirno nel far perdere le proprie tracce, ma anche intellettuale preoccupato di diffondere coi suoi manoscritti tra i contemporanei e di trasmettere alla posterità quel pensiero delle Lumières al quale hanno contribuito in maniera sostanziale testi che non si osava consegnare alle stampe oppure testi all'indice, scritti che, com'è noto, godettero di straordinaria circolazione.

Introduction (pp. 11-35), l'Autrice traccia una breve storia della fortuna critica di questo "genere» - storia che trae origine dalle intuizioni di Lanson (1912) - e delle «scoperte» che si sono susseguite fino ad oggi. Se da tempo sono studiati gli scritti più noti di questa letteratura sovversiva come ad esempio il Militaire philosophe e il Testament di Meslier, il lavoro che resta da fare sull'intero corpus, sostiene l'Autrice, è appena abbozzato. La minuziosa analisi del «caso Pinchon», delle carte, dei manoscritti, della corrispondenza, delle letture, dell'intensa attività di glossatore di questo insaziabile collezionista, fornisce senza dubbio un notevole contributo alla conoscenza di quella che resta ancora una terra incognita, proprio per la clandestinità nella quale era confinato il pensiero critico del secolo dei Lumi.

4 Nei capitoli I-X, la biografia di Pinchon è il punto di partenza per una vasta indagine sulla letteratura filosofica clandestina, sul pensiero che essa ha veicolato e sulla sua circolazione. Il IX capitolo riproduce tra l'altro l'inventario che l'avventuriero stese dei suoi libri e dei suoi manoscritti: un elenco di oltre 150 pagine che fornisce indicazioni preziose sulla cultura di Pinchon e mostra costanti ed evoluzione dei suoi interessi letterari. Sempre al corrente delle novità grazie ad una rete di fornitori ed informatori appassionati quanto lui delle idee dei philosophes, Pinchon è defmito dall'Autrice un personaggio modesto della République des Lettres, i cui dettagli biografici interessano in quanto le sue relazioni, la sua attività di collezionista e di copista, il suo ruolo di testimone «non innocente» illustrano un'epoca e un ambiente.

Il volume è corredato da un utile indice dei nomi menzionati nelle carte qui analizzate e da una bibliografia monumentale (pp. 347-409).

6 La seconda opera qui presentata è articolata in cinque insiemi («Des écrits dont la lecture est dangereuse»; «Anonymat et autobiographie intellectuelle: Robert Challe»; «Attributions et réceptions: Boulainvilliers et le curé Meslier»; «Les métamorphoses de Telliamed»; «Tactiques de Voltaire») che raccolgono 25 saggi già pubblicati dall'Autrice dal 1983 al 2001. Le carte di Pinchon hanno permesso a Geneviève Artigas-Menant, a mano a mano che procedeva nell'indagine, di fare confronti, di acquisire informazioni di rilievo sul fenomeno in generale o di fornire nuove interpretazioni di testi-faro di questa letteratura.

7 Il primo gruppo di articoli pone il problema dei limiti del corpus clandestino, un insieme di testi che presenta caratteristiche disomogenee per qualità letteraria e tendenze filosofiche, pur emanando, dai manoscritti, che lo compongono, una cultura condivisa e uno stesso approccio critico dei loro autori, nonché dei loro lettori. La coesione del corpus è assicurata, secondo l'Autrice, dal metodo e dalla finalità comune: la lotta antireligiosa. L'unità ideologica del fenomeno clandestino sta nelle tecniche messe a punto per sviluppare e diffondere l'incredulità.

8 Per quanto concerne Robert Challe, autore del primo trattato deista francese, sono le sue contraddizioni che attirano l'attenzione della studiosa. Dal suo dialogo con 
Malebranche, emerge una difficoltà insormontabile a conciliare un razionalismo alimentato dall'odio per le superstizioni, per il clero, per i dogmi e un'attrazione malcelata per forme di religione ancorate alla tradizione. Le Difficultés sur la religion sono così l'espressione di una rivolta, ma anche di un'inquietudine.

Nessuna parentela ideologica tra Boulainvilliers e Meslier, ai quali sono dedicati i tre studi della terza parte: $i$ loro testi clandestini si sono incontrati nella biblioteca di Pinchon, e qui hanno ricevuto entrambi le attenzioni del collezionista che li ha corredati di una ricca documentazione. Questo ha sollevato problemi di attribuzione e di ricezione le cui risposte sono in parte fornite dalle carte del nostro avventuriero.

10 A Telliamed di Benoît de Maillet, spetta il compito di illustrare l'elaborazione di un manoscritto e le trasformazioni subite nei trent'anni di vita clandestina, prima della sua pubblicazione. Il ruolo della riscrittura è centrale per quanto concerne la chiarezza e lo stile. Le trasformazioni sono sempre migliorative.

11 Ed ecco infme Voltaire, il suo ruolo e le sue strategie nella diffusione della philosophie attraverso testi manoscritti e opere a stampa. Pur traendo profitto dai metodi della generazione precedente, Voltaire scardina - o piuttosto capovolge - i procedimenti tipici di un «genere» (critica erudita, ricerca individuale, circolazione segreta). Se gli autori della prima metà del Settecento non avevano bisogno di palcoscenici per affermare le loro idee e la loro libertà intellettuale, Voltaire lancia proclami, anima dissensi sociali, suscita dibattiti. La propaganda voltairiana non si priva del manoscritto clandestino, ma la copia diventa adattamento e la «fabbrica di Ferney» contribuisce a rinnovare i meccanismi e a far evolvere la funzione della clandestinità, proprio in virtù della pubblicazione, ormai sempre più abbondante verso la metà del secolo, di opere nelle quali le idee dei philosophes sono esposte senza veli. Tra il «règne du manuscrit» $\mathrm{e}$ il «règne de l'imprimé» (p. 16) non c'è, secondo l'Autrice, soluzione di continuità. L'élite che leggeva manoscritti prima del 1750, diventa il pubblico di Voltaire senza che la tradizione clandestina venga meno. Il percorso indicato dal titolo del volume si compie quindi senza scosse e ostacoli.

12 La Conclusion (che reca il sottotitolo La plume et les Lumières: le manuscrit, outil de progrès) pone una serie di quesiti tra i quali uno in particolare - benché la risposta possa apparire scontata - permette alla studiosa di spiegare lo straordinario interesse di questa letteratura: «Pourquoi, à l'époque de l'imprimerie, le manuscrit est-il un fantastique outil de progrès?» (p. 352). I manoscritti filosofici clandestini, risponde l'Autrice - hanno preparato la rivoluzione intellettuale degli anni maturi dell'illuminismo, contribuendo dapprima alla formazione dei philosophes, poi alla diffusione delle loro opere a stampa condannate dalla giustizia, attraverso una rete già consolidata e in continua espansione. Il successo del manoscritto filosofico clandestino non può essere spiegato soltanto con banali motivazioni, come l'assenza della libertà di espressione, la prudenza di scrittori ed editori di testi pericolosi che temevano le conseguenze giudiziarie, i vantaggi economici... La studiosa mette in luce la creatività individuale e collettiva che si esercita nelle "operazioni» tipiche del copista: prestiti, varianti, riassunti, correzioni, commenti, revisioni, contaminazioni... L'effervescenza intellettuale e la vasta attività di elaborazione di idee scaturiscono da queste operazioni che costituiscono le vere risorse del manoscritto. In questo «chantier intellectuel, transformable à l'infini, totalement ouvert à la liberté d'expression et d'endoctrinement, à l'expérimentation des idées et à leur reproduction, [...] à la contribution des intelligences, à la répercussion des savoirs» (p. 369), si inventano 
nuove modalità di scrittura e di lettura, si mette a punto un metodo di diffusione straordinariamente efficace. È questo che, secondo l'Autrice, giustifica la sopravvivenza e l'intensificazione del fenomeno nel corso del secolo. Per questo, conclude Geneviève Artigas-Menant il manoscritto è un messaggio, un'esortazione, un dibattito a più voci che stimola il progresso del pensiero, il costituirsi di un sapere collettivo e la formazione di uno spirito critico in permanente remise en question.

13 Frutto di studi di ampio respiro, che confermano le già note competenze di Geneviève Artigas-Menant e la sua familiarità coi testi, il pensiero e gli strumenti della philosophie, i due volumi, risultato di una ricerca ventennale, apportano un contributo di tutto rilievo alla conoscenza del pensiero trasgressivo del secolo. 